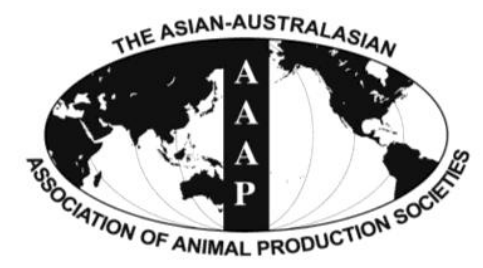

Asian Australas. J. Anim. Sci.

Vol. 26, No. 12 : 1708-1716 December 2013

http://dx.doi.org/10.5713/ajas.2013.13245

www.ajas.info

pISSN 1011-2367 elSSN 1976-5517

\title{
Relationship between the Methane Production and the CNCPS Carbohydrate Fractions of Rations with Various Concentrate/roughage Ratios Evaluated Using In vitro Incubation Technique
}

\author{
Ruilan Dong and Guangyong Zhao* \\ College of Animal Science and Technology, China Agricultural University, \\ State Key Laboratory of Animal Nutrition, Beijing 100193, China
}

\begin{abstract}
The objective of the trial was to study the relationship between the methane $\left(\mathrm{CH}_{4}\right)$ production and the Cornell Net Carbohydrate and Protein System (CNCPS) carbohydrate fractions of feeds for cattle and the suitability of CNCPS carbohydrate fractions as the dietary variables in modeling the $\mathrm{CH}_{4}$ production in rumen fermentation. Forty-five rations for cattle with the concentrate/roughage ratios of 10:90, 20:80, 30:70, 40:60, and 50:50 were formulated as feed samples. The Menke and Steingass's gas test was used for the measurement of $\mathrm{CH}_{4}$ production. The feed samples were incubated for $48 \mathrm{~h}$ and the $\mathrm{CH}_{4}$ production was analyzed using gas chromatography. Statistical analysis indicated that the $\mathrm{CH}_{4}$ production $(\mathrm{mL})$ was closely correlated with the CNCPS carbohydrate fractions (g), i.e. $\mathrm{CA}$ (sugars); $\mathrm{CB}_{1}$ (starch and pectin); $\mathrm{CB}_{2}$ (available cell wall) in a multiple linear pattern: $\mathrm{CH}_{4}=$ (89.16 \pm 14.93$) \mathrm{CA}+(124.10 \pm 13.90) \mathrm{CB}_{1}+(30.58 \pm 11.72) \mathrm{CB}_{2}+(3.28 \pm 7.19), \mathrm{R}^{2}=0.81, \mathrm{p}<0.0001, \mathrm{n}=45$. Validation of the model using 10 rations indicated that the $\mathrm{CH}_{4}$ production of the rations for cattle could accurately be predicted based on the CNCPS carbohydrate fractions. The trial indicated that the CNCPS carbohydrate fractions $\mathrm{CA}, \mathrm{CB}_{1}$ and $\mathrm{CB}_{2}$ were suitable dietary variables for predicting the $\mathrm{CH}_{4}$ production in rumen fermentation in vitro. (Key Words: Methane Production, CNCPS, Cattle, In vitro Incubation)
\end{abstract}

\section{INTRODUCTION}

To accurately predict the $\mathrm{CH}_{4}$ production in rumen fermentation is important for identifying the strategies for mitigating $\mathrm{CH}_{4}$ production from ruminants. Many studies indicated that the nutrient composition of feeds is closely correlated with the $\mathrm{CH}_{4}$ production in rumen fermentation. Since there is a correlation between the feed composition and the $\mathrm{CH}_{4}$ production, the feed composition is used to predict the $\mathrm{CH}_{4}$ production. The dietary variables including dry matter intake (DMI) (Kriss, 1930; Axelsson, 1949; Mills et al., 2003; Ellis et al., 2007), energy digestibility (Blaxter and Clapperton, 1965), digestible carbohydrates (dCHO) (Bratzler and Forbes, 1940), intakes of nonstructural carbohydrate (NSC), hemicellulose and cellulose (Moe and Tyrell, 1979) and digestible crude protein (CP), ether extract (EE), crude fibre and $\mathrm{N}$-free extract of the diets (Jentsch et al., 2007) etc. were used in $\mathrm{CH}_{4}$ predicting models. The use of different dietary variables in different

\footnotetext{
* Corresponding Author: Guangyong Zhao. Tel: +86-10-627 33379, Fax: +86-10-62733379, E-mail: zhaogy@ cau.edu.cn Submitted May 5, 2013; Accepted Jul. 2, 2013; Revised Aug. 6, 2013
}

models affected the accuracy of $\mathrm{CH}_{4}$ predicting models. Screening the dietary parameters which are easily determined and closely correlated with the $\mathrm{CH}_{4}$ production would be helpful for accurately modeling and predicting the $\mathrm{CH}_{4}$ production from ruminants.

The Cornell Net Carbohydrate and Protein System (CNCPS) divided the carbohydrates and the nitrogenous compounds of feeds into detailed fractions based on the fermentative characteristics in the rumen (Sniffen et al., 1992). Many trials indicated that the CNCPS fractions closely correlated with some indices of rumen fermentation, including the duodenal flow of microbial $\mathrm{N}$ (Offner and Sauvant, 2004) and the in situ undegraded dietary protein (UDP) for ruminants (Shannak et al., 2000) etc. Since $\mathrm{CH}_{4}$ is one of the important products of microbial fermentation of carbohydrates in the rumen, it could be speculated that the $\mathrm{CH}_{4}$ production in rumen fermentation could be closely correlated with the CNCPS carbohydrate fractions.

The objectives of the present trial were to study the relationship between the rumen $\mathrm{CH}_{4}$ production from feeds for cattle and the CNCPS carbohydrate fractions, and the suitability of the CNCPS carbohydrate fractions as dietary 
variables in modeling $\mathrm{CH}_{4}$ production in rumen fermentation.

\section{MATERIALS AND METHODS}

\section{Animals and feeding management}

Two castrated Simmental bulls, aged $1.5 \mathrm{yrs,} \mathrm{with}$ average liveweight of $372 \pm 6 \mathrm{~kg}$ and fitted with permanent rumen fistulas made of polyethylene (Beijing Jinniuweiye Science and Technology Co., Ltd., Beijing, China), were used as the donors of rumen fluid. The daily ration for the cattle included $6.0 \mathrm{~kg}$ Chinese wildrye and $2.0 \mathrm{~kg}$ concentrate mixture. The concentrate mixture was composed of $58 \%$ corn, $20 \%$ soybean meal, $18 \%$ wheat bran, $2 \%$ calcium hydrogen phosphate, $1 \%$ sodium chloride, and $1 \%$ trace element mixture. The cattle were fed twice daily at 07:00 $\mathrm{h}$ and 17:00 h, in two equal meals, and had free access to fresh drinking water. The management of the cattle was according to The Administration Regulations on Laboratory Animals (The Administrative Department of Beijing Municipal Science and Technology, 2002).

\section{Feed samples}

Air-dried feeds for cattle, milled to pass a screen with the pore size of $1 \mathrm{~mm}$, were used as the materials for formulating rations. Forty-five rations for cattle with the concentrate/roughage ratios of 10:90, 20:80, 30:70, 40:60, and 50:50 were formulated as the feed samples, of which 9 rations were formulated for each concentrate/roughage ratio. The components of the rations were shown in Table 1.

\section{In vitro incubation}

The Menke and Steingass's (1988) gas test was used for the measurement of $\mathrm{CH}_{4}$ production of feed samples. Glass syringes with a calibrated volume of $100 \mathrm{~mL}$ were used as the incubation vessels.

Two hundred $\mathrm{mL}$ of rumen fluid was taken from each cattle through the rumen fistulas $2 \mathrm{~h}$ after morning feeding. The rumen fluid from the two cattle was well mixed and immediately strained through four layers of gauze into prewarmed bottle $\left(39^{\circ} \mathrm{C}\right)$. Three hundred $\mathrm{mL}$ of rumen fluid and $600 \mathrm{~mL}$ buffer were mixed and continuously gassed with carbon dioxide. Each syringe contained $0.2000 \mathrm{~g}$ feed sample and the syringes were pre-warmed at $39^{\circ} \mathrm{C}$. Four syringes were used for each ration as replicates and three syringes without feed samples were used as the blanks for each batch of samples. Each syringe was filled with $30 \mathrm{~mL}$ rumen fluid-buffer mixture. The air in the syringes was transpired and the heads of the syringes were sealed. The syringes were kept in a water bath at $39^{\circ} \mathrm{C}$ for incubation. The total gas production of feed samples was recorded and the $\mathrm{pH}$ of incubation residue was immediately determined after incubated for $48 \mathrm{~h}$. A $5 \mathrm{~mL}$ gas sample was taken through a syringe needle connector fitted between the sampling syringe and the incubation syringe for the analysis of gas composition.

\section{Determinations and analysis}

The dry matter (DM), EE and ash of the feed samples were determined according to AOAC (1990) using the methods of no. 934.01, 920.39, and 924.05, respectively. The CP of feed samples was analyzed using the Kjeldahl method. The neutral detergent fibre (NDF) was analyzed using the method of Van Soest et al. (1991). The neutral detergent insoluble CP (NDICP) was analyzed by determination of the $\mathrm{CP}$ in the NDF residues. The acid detergent lignin was analyzed using the method of Goering and Van Soest (1970). The starch content of feed samples was determined using spectrophotometry (UV-9100, Beijing Ruili Analytical Instruments, China) after converting starch to glucose using an enzyme kit containing thermostable $\alpha$-amylase and amyloglucosidase (Megazyme International Ireland Ltd., Wicklow, Ireland; Method 996.11, AOAC, 1990).

The concentrations of $\mathrm{CH}_{4}$ and $\mathrm{CO}_{2}$ in the gas samples were analyzed using gas chromatography (TP-2060T, Beijing Beifen Tianpu Instrument Technology Co., Ltd., Beijing, China). The conditions for the analysis were as following: TCD detector, TDX-01 column, size $1 \mathrm{~m} \times 2 \mathrm{~mm}$ $\times 3 \mathrm{~mm}$, column temperature $70^{\circ} \mathrm{C}$, detector temperature $100^{\circ} \mathrm{C}$. The carrying gas was argon, with the flowing rate of $30 \mathrm{~mL} / \mathrm{min}$. The standard gas used was composed of $26.796 \% \mathrm{CH}_{4}, 65.300 \% \mathrm{CO}_{2}, 0.605 \% \mathrm{O}_{2}, 7.100 \% \mathrm{~N}_{2}$ and $0.199 \% \mathrm{H}_{2}(\mathrm{v} / \mathrm{v})$.

\section{Calculation and statistical analysis}

The CNCPS carbohydrate fractions of the rations for modeling were calculated according to Sniffen et al. (1992) and listed in Table 2.

$$
\begin{aligned}
& \mathrm{CA}=\mathrm{NSC}-\text { Starch } \\
& \mathrm{CB}_{1}=\text { Starch } \\
& \mathrm{CB}_{2}=\mathrm{NDF}-\mathrm{NDICP}-\mathrm{CC} \\
& \mathrm{CC}=\text { Lignin } \times 2.4 \\
& \mathrm{NSC}=\mathrm{CHO}-\mathrm{CB}_{2}-\mathrm{CC} \\
& \mathrm{CHO}=100-\mathrm{CP}-\text { Ash }-\mathrm{EE}
\end{aligned}
$$

Where $\mathrm{CA}$ refers to sugars; $\mathrm{CB}_{1}$, starch and pectin; $\mathrm{CB}_{2}$, available cell wall; $\mathrm{CC}$, unavailable cell wall; NSC, nonstructural carbohydrate; $\mathrm{CHO}$, carbohydrate; $\mathrm{CP}$, crude protein; NDICP, neutral detergent insoluble crude protein. 
Table 1. The components of the rations for modeling (\%, air dry basis)

\begin{tabular}{|c|c|c|c|c|c|c|c|c|c|c|c|c|c|c|}
\hline $\begin{array}{c}\text { Ration } \\
\text { no. }\end{array}$ & Corn & $\begin{array}{c}\text { Soybean } \\
\text { meal }\end{array}$ & $\begin{array}{l}\text { Wheat } \\
\text { bran }\end{array}$ & $\begin{array}{c}\text { Cottonseed } \\
\text { meal }\end{array}$ & $\begin{array}{c}\text { Rapeseed } \\
\text { meal }\end{array}$ & DDGS & $\begin{array}{l}\text { Wheat } \\
\text { middlings }\end{array}$ & $\begin{array}{l}\text { Rice } \\
\text { straw }\end{array}$ & $\begin{array}{l}\text { Corn } \\
\text { stover }\end{array}$ & $\begin{array}{l}\text { Corn } \\
\text { silage }\end{array}$ & $\begin{array}{l}\text { Wheat } \\
\text { straw }\end{array}$ & $\begin{array}{l}\text { Millet } \\
\text { straw }\end{array}$ & $\begin{array}{l}\text { Chinese } \\
\text { wildrye }\end{array}$ & $\begin{array}{c}\text { Concentrate/ } \\
\text { roughage } \\
\text { ratio }\end{array}$ \\
\hline 1 & 28.5 & 11.5 & 10.0 & - & - & - & - & 50 & - & - & - & - & - & $50: 50$ \\
\hline 2 & 22.8 & 9.2 & 8.0 & - & - & - & - & 60 & - & - & - & - & - & $40: 60$ \\
\hline 3 & 17.1 & 6.9 & 6.0 & - & - & - & - & 70 & - & - & - & - & - & $30: 70$ \\
\hline 4 & 11.4 & 4.6 & 4.0 & - & - & - & - & 80 & - & - & - & - & - & $20: 80$ \\
\hline 5 & 28.5 & 11.5 & 10.0 & - & - & - & - & - & - & 25 & - & 25 & - & $50: 50$ \\
\hline 6 & 22.8 & 9.2 & 8.0 & - & - & - & - & - & - & 39 & - & 21 & - & $40: 60$ \\
\hline 7 & 17.1 & 6.9 & 6.0 & - & - & - & - & - & - & 56 & - & 14 & - & $30: 70$ \\
\hline 8 & 5.7 & 2.3 & 2.0 & - & - & - & - & - & - & 45 & - & 45 & - & $10: 90$ \\
\hline 9 & 27.5 & - & 9.5 & 13.0 & - & - & - & - & 50 & - & - & - & - & $50: 50$ \\
\hline 10 & 22.0 & - & 7.6 & 10.4 & - & - & - & - & 60 & - & - & - & - & $40: 60$ \\
\hline 11 & 11.0 & - & 3.8 & 5.2 & - & - & - & - & 80 & - & - & - & - & $20: 80$ \\
\hline 12 & 5.5 & - & 1.9 & 2.6 & - & - & - & - & 90 & - & - & - & - & $10: 90$ \\
\hline 13 & 26.5 & 7.5 & 9.5 & - & 6.5 & - & - & - & - & 50 & - & - & - & $50: 50$ \\
\hline 14 & 15.9 & 4.5 & 5.7 & - & 3.9 & - & - & - & - & 70 & - & - & - & $30: 70$ \\
\hline 15 & 10.6 & 3.0 & 3.8 & - & 2.6 & - & - & - & - & 80 & - & - & - & $20: 80$ \\
\hline 16 & 5.3 & 1.5 & 1.9 & - & 1.3 & - & - & - & - & 90 & - & - & - & $10: 90$ \\
\hline 17 & 18.8 & 6.0 & 6.0 & 4.0 & - & 2.4 & 2.8 & - & - & - & - & - & 60 & $40: 60$ \\
\hline 18 & 14.1 & 4.5 & 4.5 & 3.0 & - & 1.8 & 2.1 & - & - & - & - & - & 70 & $30: 70$ \\
\hline 19 & 9.4 & 3.0 & 3.0 & 2.0 & - & 1.2 & 1.4 & - & - & - & - & - & 80 & $20: 80$ \\
\hline 20 & 4.7 & 1.5 & 1.5 & 1.0 & - & 0.6 & 0.7 & - & - & - & - & - & 90 & $10: 90$ \\
\hline 21 & 23.5 & 7.5 & 7.5 & 5.0 & - & 3.0 & 3.5 & - & 25 & - & 25 & - & - & $50: 50$ \\
\hline 22 & 18.8 & 6.0 & 6.0 & 4.0 & - & 2.4 & 2.8 & - & 39 & - & 21 & - & - & $40: 60$ \\
\hline 23 & 14.1 & 4.5 & 4.5 & 3.0 & - & 1.8 & 2.1 & - & 56 & - & 14 & - & - & $30: 70$ \\
\hline 24 & 9.4 & 3.0 & 3.0 & 2.0 & - & 1.2 & 1.4 & - & 60 & - & 20 & - & - & $20: 80$ \\
\hline 25 & 26.0 & - & 9.0 & 7.5 & 7.5 & - & - & - & - & - & - & 50 & - & $50: 50$ \\
\hline 26 & 20.8 & - & 7.2 & 6.0 & 6.0 & - & - & - & - & - & - & 60 & - & $40: 60$ \\
\hline 27 & 15.6 & - & 5.4 & 4.5 & 4.5 & - & - & - & - & - & - & 70 & - & $30: 70$ \\
\hline 28 & 5.2 & - & 1.8 & 1.5 & 1.5 & - & - & - & - & - & - & 90 & - & $10: 90$ \\
\hline 29 & 26.0 & - & 9.0 & 7.5 & 7.5 & - & - & - & - & - & 25 & 25 & - & $50: 50$ \\
\hline 30 & 20.8 & - & 7.2 & 6.0 & 6.0 & - & - & - & - & - & 39 & 21 & - & $40: 60$ \\
\hline 31 & 10.4 & - & 3.6 & 3.0 & 3.0 & - & - & - & - & - & 60 & 20 & - & $20: 80$ \\
\hline 32 & 5.2 & - & 1.8 & 1.5 & 1.5 & - & - & - & - & - & 45 & 45 & - & $10: 90$ \\
\hline 33 & 25.0 & 7.5 & 8.5 & 5.0 & 4.0 & - & - & - & - & - & 50 & - & - & $50: 50$ \\
\hline 34 & 15.0 & 4.5 & 5.1 & 3.0 & 2.4 & - & - & - & - & - & 70 & - & - & $30: 70$ \\
\hline 35 & 10.0 & 3.0 & 3.4 & 2.0 & 1.6 & - & - & - & - & - & 80 & - & - & $20: 80$ \\
\hline 36 & 5.0 & 1.5 & 1.7 & 1.0 & 0.8 & - & - & - & - & - & 90 & - & - & $10: 90$ \\
\hline 37 & 20.0 & 6.0 & 6.8 & 4.0 & 3.2 & - & - & - & - & 20 & 30 & - & 10 & $40: 60$ \\
\hline 38 & 15.0 & 4.5 & 5.1 & 3.0 & 2.4 & - & - & - & - & 35 & 21 & - & 14 & $30: 70$ \\
\hline 39 & 10.0 & 3.0 & 3.4 & 2.0 & 1.6 & - & - & - & - & 52 & 14 & - & 14 & $20: 80$ \\
\hline 40 & 5.0 & 1.5 & 1.7 & 1.0 & 0.8 & - & - & - & - & 30 & 30 & - & 30 & $10: 90$ \\
\hline 41 & 27.0 & - & 8.0 & - & 15.0 & - & - & - & 25 & - & 25 & - & - & $50: 50$ \\
\hline 42 & 21.6 & - & 6.4 & - & 12.0 & - & - & - & 39 & - & 21 & - & - & $40: 60$ \\
\hline 43 & 16.2 & - & 4.8 & - & 9.0 & - & - & - & 56 & - & 14 & - & - & $30: 70$ \\
\hline 44 & 10.8 & - & 3.2 & - & 6.0 & - & - & - & 60 & - & 20 & - & - & $20: 80$ \\
\hline 45 & 5.4 & - & 1.6 & - & 3.0 & - & - & - & 45 & - & 45 & - & - & 10:90 \\
\hline
\end{tabular}

The unit for all the CNCPS fractions is \% DM.

The $\mathrm{CH}_{4}, \mathrm{CO}_{2}$ or total gas production of feed samples $(\mathrm{mL})$ was calculated as following:

$$
\mathrm{Y}_{\text {sample }}=\mathrm{Y}_{\text {total }}-\mathrm{Y}_{\text {blank }}
$$

Where, $\mathrm{Y}_{\text {sample }}$ refers to the $\mathrm{CH}_{4}$ production of feed sample in $48 \mathrm{~h} ; \mathrm{Y}_{\text {total }}$, the $\mathrm{CH}_{4}, \mathrm{CO}_{2}$ or total gas production of incubation in $48 \mathrm{~h}$; $\mathrm{Y}_{\text {blank }}$, the $\mathrm{CH}_{4}, \mathrm{CO}_{2}$ or total gas production of the blank in $48 \mathrm{~h}$. The $\mathrm{CH}_{4}, \mathrm{CO}_{2}$ and total gas production and the $\mathrm{pH}$ for modeling were listed in Table 3 .

The regression relationship between the $\mathrm{CH}_{4}, \mathrm{CO}_{2}$ and total gas production $(\mathrm{mL})$ and the CNCPS carbohydrate fractions ( $\mathrm{g}$ ) was analyzed using the following equation:

$$
y=b_{1}(\mathrm{CA})+b_{2}\left(\mathrm{CB}_{1}\right)+b_{3}\left(\mathrm{CB}_{2}\right)+a
$$

Where, $y$ refers to the $\mathrm{CH}_{4}, \mathrm{CO}_{2}$ or total gas production; 
Table 2. The CP and CNCPS carbohydrate fractions of the rations for modeling (\% DM)

\begin{tabular}{|c|c|c|c|c|c|c|c|}
\hline \multirow{2}{*}{$\begin{array}{c}\text { Ration } \\
\text { no. }\end{array}$} & \multirow{2}{*}{$\mathrm{CP}$} & \multirow{2}{*}{ Carbohydrates } & \multicolumn{4}{|c|}{ Carbohydrate fractions } & \multirow{2}{*}{ NSC } \\
\hline & & & $\mathrm{CA}$ & $\mathrm{CB}_{1}$ & $\mathrm{CB}_{2}$ & $\mathrm{CC}$ & \\
\hline 1 & 12.79 & 77.34 & 10.03 & 19.56 & 39.97 & 7.75 & 29.60 \\
\hline 2 & 11.22 & 77.95 & 9.45 & 15.94 & 43.96 & 8.57 & 25.40 \\
\hline 3 & 9.64 & 78.56 & 8.87 & 12.33 & 47.95 & 9.40 & 21.20 \\
\hline 4 & 8.07 & 79.17 & 8.29 & 8.71 & 51.93 & 10.23 & 17.00 \\
\hline 5 & 12.81 & 80.68 & 14.41 & 19.15 & 38.13 & 8.96 & 33.56 \\
\hline 6 & 11.20 & 82.03 & 13.99 & 15.47 & 43.02 & 9.51 & 29.46 \\
\hline 7 & 9.58 & 83.40 & 13.33 & 11.81 & 48.34 & 9.90 & 25.14 \\
\hline 8 & 6.54 & 85.78 & 15.59 & 4.35 & 52.61 & 13.24 & 19.93 \\
\hline 9 & 15.04 & 76.89 & 12.74 & 18.38 & 36.27 & 9.48 & 31.12 \\
\hline 10 & 13.96 & 77.40 & 13.26 & 14.82 & 39.78 & 9.51 & 28.08 \\
\hline 11 & 11.80 & 78.41 & 14.28 & 7.71 & 46.82 & 9.58 & 21.99 \\
\hline 12 & 10.72 & 78.91 & 14.80 & 4.16 & 50.34 & 9.62 & 18.95 \\
\hline 13 & 13.14 & 79.92 & 12.01 & 17.92 & 40.90 & 9.06 & 29.93 \\
\hline 14 & 9.79 & 82.93 & 11.98 & 11.06 & 49.86 & 10.02 & 23.04 \\
\hline 15 & 8.11 & 84.44 & 11.96 & 7.63 & 54.34 & 10.49 & 19.59 \\
\hline 16 & 6.44 & 85.94 & 11.94 & 4.21 & 58.82 & 10.97 & 16.15 \\
\hline 17 & 12.59 & 80.35 & 14.40 & 13.92 & 37.91 & 14.10 & 28.32 \\
\hline 18 & 10.98 & 81.69 & 14.57 & 10.53 & 41.20 & 15.38 & 25.10 \\
\hline 19 & 9.37 & 83.03 & 14.74 & 7.14 & 44.48 & 16.66 & 21.88 \\
\hline 20 & 7.75 & 84.37 & 14.92 & 3.74 & 47.77 & 17.94 & 18.66 \\
\hline 21 & 14.81 & 77.33 & 11.78 & 17.39 & 35.99 & 12.15 & 29.17 \\
\hline 22 & 13.73 & 77.81 & 12.39 & 14.03 & 39.56 & 11.81 & 26.41 \\
\hline 23 & 12.79 & 78.11 & 13.30 & 10.67 & 43.14 & 10.98 & 23.97 \\
\hline 24 & 11.25 & 79.17 & 12.88 & 7.29 & 46.69 & 12.29 & 20.17 \\
\hline 25 & 13.24 & 79.54 & 15.02 & 17.36 & 33.35 & 13.78 & 32.38 \\
\hline 26 & 11.63 & 80.97 & 15.98 & 13.99 & 36.51 & 14.46 & 29.97 \\
\hline 27 & 10.01 & 82.39 & 16.95 & 10.62 & 39.67 & 15.13 & 27.57 \\
\hline 28 & 6.79 & 85.24 & 18.88 & 3.88 & 46.00 & 16.48 & 22.76 \\
\hline 29 & 13.21 & 79.19 & 11.34 & 17.32 & 34.48 & 16.02 & 28.66 \\
\hline 30 & 11.58 & 80.42 & 10.24 & 13.94 & 38.28 & 17.94 & 24.18 \\
\hline 31 & 8.33 & 82.97 & 9.07 & 7.17 & 45.55 & 21.17 & 16.24 \\
\hline 32 & 6.74 & 84.61 & 12.25 & 3.82 & 48.03 & 20.50 & 16.07 \\
\hline 33 & 14.19 & 78.02 & 8.92 & 16.66 & 35.59 & 16.83 & 25.59 \\
\hline 34 & 10.54 & 80.92 & 7.40 & 10.15 & 42.82 & 20.53 & 17.55 \\
\hline 35 & 8.71 & 82.37 & 6.63 & 6.89 & 46.44 & 22.38 & 13.53 \\
\hline 36 & 6.89 & 83.81 & 5.87 & 3.64 & 50.06 & 24.24 & 9.51 \\
\hline 37 & 12.41 & 79.95 & 10.52 & 13.48 & 40.87 & 15.06 & 24.00 \\
\hline 38 & 10.58 & 81.75 & 11.18 & 10.29 & 45.82 & 14.45 & 21.46 \\
\hline 39 & 8.71 & 83.57 & 11.57 & 7.10 & 51.08 & 13.81 & 18.67 \\
\hline 40 & 7.12 & 84.60 & 10.91 & 3.75 & 52.16 & 17.78 & 14.66 \\
\hline 41 & 13.95 & 77.32 & 9.85 & 17.78 & 35.44 & 14.22 & 27.63 \\
\hline 42 & 13.04 & 77.80 & 10.84 & 14.34 & 39.12 & 13.47 & 25.18 \\
\hline 43 & 12.27 & 78.10 & 12.14 & 10.91 & 42.81 & 12.23 & 23.04 \\
\hline 44 & 10.90 & 79.16 & 12.11 & 7.45 & 46.47 & 13.12 & 19.55 \\
\hline 45 & 8.67 & 81.34 & 10.14 & 3.95 & 50.10 & 17.14 & 14.09 \\
\hline
\end{tabular}

$a$ refers to a constant; $b_{1}, b_{2}$, and $b_{3}$ refer to coefficients.

Ten rations with the same range of concentrate/ roughage ratio as that of the 45 rations for modeling with two rations for each ratio were formulated to validate the relationships between the $\mathrm{CH}_{4}, \mathrm{CO}_{2}$ and total gas production and the CNCPS carbohydrate fractions. The components, the nutrient composition and the $\mathrm{CH}_{4}, \mathrm{CO}_{2}$ and total gas production of the rations for validation were listed 
Table 3. The $\mathrm{CH}_{4}, \mathrm{CO}_{2}$ and total gas production and $\mathrm{pH}$ of the rations for modeling

\begin{tabular}{|c|c|c|c|c|}
\hline $\begin{array}{l}\text { Ration } \\
\text { no. }\end{array}$ & $\begin{array}{c}\mathrm{CH}_{4} \\
(\mathrm{~mL} / \mathrm{g} \mathrm{DM})\end{array}$ & $\begin{array}{c}\mathrm{CO}_{2} \\
(\mathrm{~mL} / \mathrm{g} \mathrm{DM})\end{array}$ & $\begin{array}{l}\text { Total gas } \\
(\mathrm{mL} / \mathrm{g} \mathrm{DM})\end{array}$ & $\mathrm{pH}$ \\
\hline 1 & $50 \pm 0$ & $189 \pm 2$ & $248 \pm 2$ & $6.63 \pm 0.00$ \\
\hline 2 & $47 \pm 1$ & $186 \pm 3$ & $241 \pm 3$ & $6.61 \pm 0.01$ \\
\hline 3 & $45 \pm 1$ & $174 \pm 6$ & $227 \pm 6$ & $6.63 \pm 0.01$ \\
\hline 4 & $40 \pm 0$ & $155 \pm 1$ & $202 \pm 1$ & $6.71 \pm 0.01$ \\
\hline 5 & $49 \pm 1$ & $213 \pm 1$ & $272 \pm 1$ & $6.58 \pm 0.00$ \\
\hline 6 & $47 \pm 1$ & $209 \pm 3$ & $266 \pm 3$ & $6.58 \pm 0.00$ \\
\hline 7 & $42 \pm 1$ & $198 \pm 3$ & $250 \pm 3$ & $6.58 \pm 0.01$ \\
\hline 8 & $37 \pm 0$ & $185 \pm 3$ & $232 \pm 5$ & $6.63 \pm 0.02$ \\
\hline 9 & $51 \pm 1$ & $172 \pm 3$ & $240 \pm 3$ & $6.66 \pm 0.01$ \\
\hline 10 & $48 \pm 0$ & $150 \pm 6$ & $214 \pm 6$ & $6.74 \pm 0.01$ \\
\hline 11 & $37 \pm 1$ & $160 \pm 3$ & $207 \pm 4$ & $6.77 \pm 0.00$ \\
\hline 12 & $33 \pm 1$ & $143 \pm 6$ & $186 \pm 5$ & $6.78 \pm 0.01$ \\
\hline 13 & $49 \pm 1$ & $218 \pm 4$ & $281 \pm 2$ & $6.51 \pm 0.00$ \\
\hline 14 & $45 \pm 1$ & $216 \pm 3$ & $272 \pm 2$ & $6.57 \pm 0.00$ \\
\hline 15 & $40 \pm 1$ & $173 \pm 5$ & $223 \pm 5$ & $6.55 \pm 0.01$ \\
\hline 16 & $37 \pm 1$ & $162 \pm 11$ & $208 \pm 11$ & $6.57 \pm 0.00$ \\
\hline 17 & $47 \pm 1$ & $175 \pm 3$ & $235 \pm 4$ & $6.47 \pm 0.02$ \\
\hline 18 & $43 \pm 1$ & $156 \pm 3$ & $211 \pm 2$ & $6.51 \pm 0.02$ \\
\hline 19 & $40 \pm 1$ & $144 \pm 4$ & $195 \pm 4$ & $6.55 \pm 0.01$ \\
\hline 20 & $36 \pm 0$ & $135 \pm 0$ & $181 \pm 0$ & $6.58 \pm 0.00$ \\
\hline 21 & $45 \pm 1$ & $177 \pm 2$ & $232 \pm 3$ & $6.64 \pm 0.02$ \\
\hline 22 & $43 \pm 1$ & $173 \pm 2$ & $225 \pm 1$ & $6.56 \pm 0.01$ \\
\hline 23 & $39 \pm 1$ & $166 \pm 2$ & $213 \pm 4$ & $6.59 \pm 0.01$ \\
\hline 24 & $37 \pm 1$ & $151 \pm 2$ & $195 \pm 3$ & $6.59 \pm 0.01$ \\
\hline 25 & $52 \pm 1$ & $194 \pm 5$ & $260 \pm 5$ & $6.73 \pm 0.00$ \\
\hline 26 & $51 \pm 0$ & $182 \pm 1$ & $245 \pm 0$ & $6.75 \pm 0.00$ \\
\hline 27 & $48 \pm 0$ & $161 \pm 8$ & $219 \pm 9$ & $6.69 \pm 0.01$ \\
\hline 28 & $41 \pm 0$ & $155 \pm 11$ & $191 \pm 14$ & $6.77 \pm 0.00$ \\
\hline 29 & $42 \pm 1$ & $163 \pm 3$ & $214 \pm 3$ & $6.61 \pm 0.01$ \\
\hline 30 & $39 \pm 1$ & $162 \pm 4$ & $210 \pm 4$ & $6.66 \pm 0.01$ \\
\hline 31 & $33 \pm 1$ & $135 \pm 2$ & $176 \pm 2$ & $6.75 \pm 0.01$ \\
\hline 32 & $30 \pm 1$ & $127 \pm 1$ & $165 \pm 1$ & $6.74 \pm 0.00$ \\
\hline 33 & $43 \pm 1$ & $135 \pm 3$ & $191 \pm 4$ & $6.65 \pm 0.00$ \\
\hline 34 & $36 \pm 1$ & $128 \pm 11$ & $179 \pm 10$ & $6.69 \pm 0.01$ \\
\hline 35 & $36 \pm 2$ & $123 \pm 1$ & $173 \pm 3$ & $6.71 \pm 0.01$ \\
\hline 36 & $30 \pm 1$ & $126 \pm 5$ & $167 \pm 5$ & $6.69 \pm 0.01$ \\
\hline 37 & $43 \pm 1$ & $192 \pm 7$ & $254 \pm 7$ & $6.69 \pm 0.00$ \\
\hline 38 & $38 \pm 1$ & $180 \pm 4$ & $244 \pm 2$ & $6.71 \pm 0.00$ \\
\hline 39 & $38 \pm 1$ & $171 \pm 3$ & $226 \pm 2$ & $6.74 \pm 0.00$ \\
\hline 40 & $34 \pm 1$ & $137 \pm 2$ & $189 \pm 4$ & $6.77 \pm 0.00$ \\
\hline 41 & $39 \pm 0$ & $171 \pm 8$ & $219 \pm 9$ & $6.58 \pm 0.01$ \\
\hline 42 & $39 \pm 0$ & $168 \pm 2$ & $220 \pm 4$ & $6.49 \pm 0.01$ \\
\hline 43 & $39 \pm 1$ & $160 \pm 8$ & $208 \pm 8$ & $6.57 \pm 0.03$ \\
\hline 44 & $37 \pm 1$ & $158 \pm 8$ & $206 \pm 7$ & $6.54 \pm 0.00$ \\
\hline 45 & $34 \pm 1$ & $141 \pm 5$ & $183 \pm 5$ & $6.57 \pm 0.04$ \\
\hline
\end{tabular}

Values were presented as mean \pm standard error (SE). in Table 4, 5 and 6, respectively.

The accuracy of the multiple regression relationships for predicting the $\mathrm{CH}_{4}, \mathrm{CO}_{2}$ and total gas production was evaluated in three ways. The observed and the predicted $\mathrm{CH}_{4}, \mathrm{CO}_{2}$ and total gas production were compared using the paired $t$-test; the relationships between the observed and the predicted $\mathrm{CH}_{4}, \mathrm{CO}_{2}$ and total gas production were analysed using the equation:

$$
y=b x+a
$$

Where, $x$ refers to the observed $\mathrm{CH}_{4}, \mathrm{CO}_{2}$ or total gas production, $\mathrm{mL} / \mathrm{g} \mathrm{DM} ; y$ refers to the predicted $\mathrm{CH}_{4}, \mathrm{CO}_{2}$ or total gas production, $\mathrm{mL} / \mathrm{g} \mathrm{DM}$; The root mean square prediction error (RMSPE) between the observed and the predicted $\mathrm{CH}_{4}, \mathrm{CO}_{2}$ or total gas production was also calculated for evaluating the multiple regression relationship established in the trial. The RMSPE was calculated as:

$$
\text { Mean square prediction error }(\mathrm{MSPE})=\sum_{i=1}^{n}(\mathrm{Oi}-\mathrm{Pi})^{2} / n
$$

RMSPE $\%=\mathrm{MSPE}^{1 / 2} /$ average observed value $\times 100$

Where, $i=1,2, \ldots, n ; O_{i}$ refers to the observed value; $P_{i}$, the predicted value; $n$, the number of determinations. RMSPE\%, the percentage of the prediction error/the average observed value.

The SAS Statistical Package 9.2 (SAS Institute Inc., Cary, NC, USA, 2008) was used for the statistical analysis of the trial. The prediction equations were developed using the PROC GLMSELECT Procedure. All the independent variables included in the equations were selected using the stepwise regression analysis by deleting non-significant variables ( $p>0.05$ ). The PROC REG Procedure was used for the analysis of the relationship between the observed and the predicted values.

\section{RESULTS}

\section{In vitro incubation}

At the end of in vitro incubation for $48 \mathrm{~h}$, the $\mathrm{pH}$ value of the incubation residue was within the range of 6.40 to 6.80 , and the microscopic check indicated that the rumen microorganisms were active.

Relationships between the $\mathrm{CH}_{4}, \mathrm{CO}_{2}$, and total gas production and the CNCPS carbohydrate fractions

The CNCPS carbohydrate fractions of the mixed rations and the $\mathrm{CH}_{4}$ production were shown in Table 2 and 3.

Significant multiple linear regression relationships were found between the $\mathrm{CH}_{4}, \mathrm{CO}_{2}$ and total gas production $(\mathrm{mL})$ 
Table 4. The components of the rations for validation (\%, air dry basis)

\begin{tabular}{|c|c|c|c|c|c|c|c|c|c|c|c|c|c|c|}
\hline $\begin{array}{l}\text { Ration } \\
\text { no. }\end{array}$ & Corn & $\begin{array}{c}\text { Soybean } \\
\text { meal }\end{array}$ & $\begin{array}{l}\text { Wheat } \\
\text { bran }\end{array}$ & $\begin{array}{c}\text { Cottonseed } \\
\text { meal }\end{array}$ & $\begin{array}{c}\text { Rapeseed } \\
\text { meal }\end{array}$ & DDGS & $\begin{array}{l}\text { Wheat } \\
\text { middlings }\end{array}$ & $\begin{array}{l}\text { Rice } \\
\text { straw }\end{array}$ & $\begin{array}{l}\text { Corn } \\
\text { stover }\end{array}$ & $\begin{array}{l}\text { Corn } \\
\text { silage }\end{array}$ & $\begin{array}{l}\text { Wheat } \\
\text { straw }\end{array}$ & $\begin{array}{l}\text { Millet } \\
\text { straw }\end{array}$ & $\begin{array}{l}\text { Chinese } \\
\text { wildrye }\end{array}$ & $\begin{array}{c}\text { Concentrate/ } \\
\text { roughage } \\
\text { ratio }\end{array}$ \\
\hline 1 & 5.7 & 2.3 & 2.0 & - & - & - & - & 90 & - & - & - & - & - & $10: 90$ \\
\hline 2 & 11.4 & 4.6 & 4.0 & - & - & - & - & - & - & 60 & - & 20 & - & $20: 80$ \\
\hline 3 & 16.5 & - & 5.7 & 7.8 & - & - & - & - & 70 & - & - & - & - & $30: 70$ \\
\hline 4 & 21.2 & 6.0 & 7.6 & - & 5.2 & - & - & - & - & 60 & - & - & - & $40: 60$ \\
\hline 5 & 23.5 & 7.5 & 7.5 & 5.0 & - & 3.0 & 3.5 & - & - & - & - & - & 50 & $50: 50$ \\
\hline 6 & 4.7 & 1.5 & 1.5 & 1.0 & - & 0.6 & 0.7 & - & 45 & - & 45 & - & - & $10: 90$ \\
\hline 7 & 10.4 & - & 3.6 & 3.0 & 3.0 & - & - & - & - & - & - & 80 & - & $20: 80$ \\
\hline 8 & 15.6 & - & 5.4 & 4.5 & 4.5 & - & - & - & - & - & 56 & 14 & - & $30: 70$ \\
\hline 9 & 20.0 & 6.0 & 6.8 & 4.0 & 3.2 & - & - & - & - & - & 60 & - & - & $40: 60$ \\
\hline 10 & 25.0 & 7.5 & 8.5 & 5.0 & 4.0 & - & - & - & - & 30 & 10 & - & 10 & $50: 50$ \\
\hline
\end{tabular}

DDGS refers to dried distiller's grains with solubles.

Table 5. The CP and CNCPS carbohydrate fractions of the rations for validating the model (\% DM)

\begin{tabular}{cccrrrrr}
\hline \multirow{2}{*}{$\begin{array}{c}\text { Ration } \\
\text { no. }\end{array}$} & \multirow{2}{*}{$\mathrm{CP}$} & \multirow{2}{*}{ Carbohydrates } & \multicolumn{3}{c}{ Carbohydrate fractions } & \multirow{2}{*}{ NSC } \\
\cline { 4 - 7 } & & & $\mathrm{CA}$ & $\mathrm{CB}_{1}$ & $\mathrm{CB}_{2}$ & $\mathrm{CC}$ & \\
\hline 1 & 6.50 & 79.78 & 7.71 & 5.09 & 55.92 & 11.05 & 12.80 \\
2 & 8.02 & 84.66 & 13.71 & 8.10 & 51.82 & 11.02 & 21.81 \\
3 & 12.88 & 77.90 & 13.77 & 11.27 & 43.30 & 9.55 & 25.04 \\
4 & 11.46 & 81.42 & 11.99 & 14.49 & 45.38 & 9.54 & 26.48 \\
5 & 14.20 & 79.02 & 14.22 & 17.32 & 34.63 & 12.82 & 31.54 \\
6 & 8.84 & 81.34 & 10.52 & 3.87 & 50.21 & 16.73 & 14.39 \\
7 & 8.40 & 83.82 & 17.91 & 7.25 & 42.84 & 15.81 & 25.16 \\
8 & 9.95 & 81.60 & 8.70 & 10.54 & 42.21 & 20.14 & 19.24 \\
9 & 12.36 & 79.47 & 8.16 & 13.41 & 39.20 & 18.68 & 21.57 \\
10 & 14.20 & 78.73 & 11.96 & 16.78 & 38.21 & 11.75 & 28.74 \\
\hline
\end{tabular}

and the CNCPS carbohydrate fractions $\mathrm{CA}, \mathrm{CB}_{1}$ and $\mathrm{CB}_{2}$ (g).

$$
\begin{aligned}
\mathrm{CH}_{4}= & (89.16 \pm 14.93) \mathrm{CA}+(124.10 \pm 13.90) \mathrm{CB}_{1} \\
& +(30.58 \pm 11.72) \mathrm{CB}_{2}+(3.28 \pm 7.19) \\
\mathrm{R}^{2}= & 0.81, \mathrm{n}=45, \mathrm{p}<0.0001 \\
\mathrm{CO}_{2}= & (435.58 \pm 69.51) \mathrm{CA}+(707.76 \pm 64.69) \mathrm{CB}_{1} \\
& +(410.42 \pm 54.58) \mathrm{CB}_{2}-(145.17 \pm 33.49)
\end{aligned}
$$

$\mathrm{R}^{2}=0.78, \mathrm{n}=45, \mathrm{p}<0.0001$

(Equation II)

Total gas $=(486.04 \pm 78.87) \mathrm{CA}+(845.98 \pm 73.41) \mathrm{CB}_{1}$ $+(439.20 \pm 61.94) \mathrm{CB}_{2}-(126.92 \pm 38.01)$

$\mathrm{R}^{2}=0.80, \mathrm{n}=45, \mathrm{p}<0.0001$

(Equation III)

Validation of the equations between the $\mathrm{CH}_{4}, \mathrm{CO}_{2}$ and

\begin{tabular}{|c|c|c|c|c|c|c|c|}
\hline \multirow{2}{*}{$\begin{array}{l}\text { Ration } \\
\text { no. }\end{array}$} & \multicolumn{2}{|c|}{$\mathrm{CH}_{4}(\mathrm{~mL} / \mathrm{g} \mathrm{DM})$} & \multicolumn{2}{|c|}{$\mathrm{CO}_{2}(\mathrm{~mL} / \mathrm{g} \mathrm{DM})$} & \multicolumn{2}{|c|}{ Total gas (mL/g DM) } & \multirow{2}{*}{$\mathrm{pH}$} \\
\hline & Observed & Predicted & Observed & Predicted & Observed & Predicted & \\
\hline 1 & $35 \pm 1$ & 34 & $142 \pm 3$ & 154 & $183 \pm 3$ & 199 & $6.69 \pm 0.01$ \\
\hline 2 & $42 \pm 1$ & 42 & $185 \pm 3$ & 184 & $237 \pm 2$ & 236 & $6.62 \pm 0.01$ \\
\hline 3 & $40 \pm 0$ & 43 & $167 \pm 0$ & 172 & $216 \pm 0$ & 225 & $6.76 \pm 0.01$ \\
\hline 4 & $46 \pm 1$ & 46 & $224 \pm 5$ & 196 & $282 \pm 4$ & 253 & $6.50 \pm 0.01$ \\
\hline 5 & $50 \pm 0$ & 48 & $177 \pm 2$ & 181 & $242 \pm 2$ & 241 & $6.46 \pm 0.01$ \\
\hline 6 & $30 \pm 0$ & 33 & $151 \pm 7$ & 134 & $187 \pm 8$ & 177 & $6.65 \pm 0.01$ \\
\hline 7 & $41 \pm 1$ & 41 & $176 \pm 21$ & 160 & $214 \pm 6$ & 210 & $6.78 \pm 0.01$ \\
\hline 8 & $36 \pm 0$ & 37 & $150 \pm 3$ & 140 & $195 \pm 4$ & 190 & $6.71 \pm 0.01$ \\
\hline 9 & $39 \pm 1$ & 39 & $142 \pm 11$ & 146 & $196 \pm 10$ & 198 & $6.71 \pm 0.01$ \\
\hline 10 & $47 \pm 1$ & 47 & $200 \pm 2$ & 182 & $261 \pm 2$ & 241 & $6.62 \pm 0.01$ \\
\hline
\end{tabular}
total gas production and the CNCPS carbohydrate fractions

Paired $t$-test showed that no difference was found

Table 6. The $\mathrm{CH}_{4}, \mathrm{CO}_{2}$ and total gas production and $\mathrm{pH}$ of the rations for validating the models 


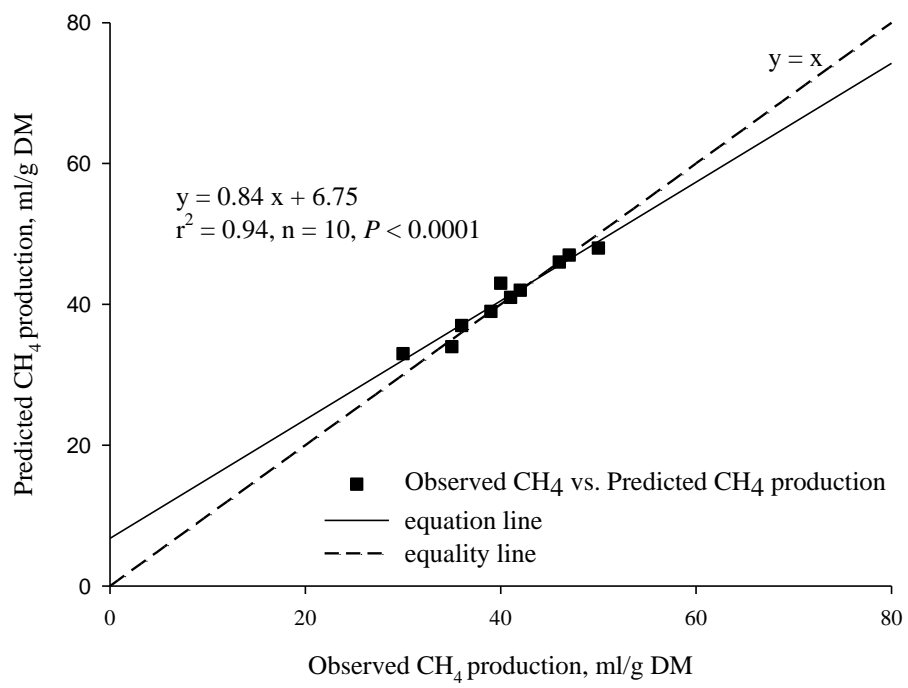

Figure 1. Relationship between the observed vs the predicted $\mathrm{CH}_{4}$ production.

between the observed and the predicted $\mathrm{CH}_{4}, \mathrm{CO}_{2}$ and total gas production based on the Equation I $(\mathrm{p}=0.443)$, Equation II $(\mathrm{p}=0.150)$ and Equation III $(\mathrm{p}=0.326)$, respectively. Significant linear regression relationship was found between the observed and the predicted $\mathrm{CH}_{4}$ production based on Equation $\mathrm{I}\left(\mathrm{R}^{2}=0.94, \mathrm{p}<0.0001, \mathrm{n}=\right.$ 10, Figure 1), between the observed and the predicted $\mathrm{CO}_{2}$ production based on Equation II $\left(\mathrm{R}^{2}=0.77, \mathrm{p}=0.0008, \mathrm{n}=\right.$ 10, Figure 2) and between the observed and the predicted total gas production based on Equation III $\left(\mathrm{R}^{2}=0.87, \mathrm{p}<\right.$ $0.0001, \mathrm{n}=10$, Figure 3). The RMSPE\% of Equations I, II, and III was found to be $3.82 \%, 8.16 \%$ and $5.93 \%$, respectively.

\section{DISCUSSION}

\section{Measurement of gas production}

The normal $\mathrm{pH}$ range and the active rumen microorganisms in the incubation residues at the end of incubation indicated that the simulation of rumen fermentation using the Hohenheim gas test (Menke and Steingass, 1988) was successful. Since the highest predictive value for the in vivo digestibility of feed was obtained after 45 to $52 \mathrm{~h}$ of in vitro fermentation (Prasad et al., 1994; Liu et al., 2002), the gas production of the rations during the $48 \mathrm{~h}$ in vitro incubation was believed to be close to that of the actual rumen fermentation.

Soe et al. (2009) reported that the in vitro gas production of ruminant feeds abundant in $\mathrm{CP}$ such as soybean meal (CP 51.3\% DM) and cell mass from lysine production (CMLP) (CP 72.3\% DM) was significantly lower than the theoretical value, indicating that the nitrogenous compounds of the feeds interfered with the acid-base reaction, increased the $\mathrm{pH}$ and reduced the indirect gas production. In this case, the in vitro gas test might be not a suitable technique for measuring the gas

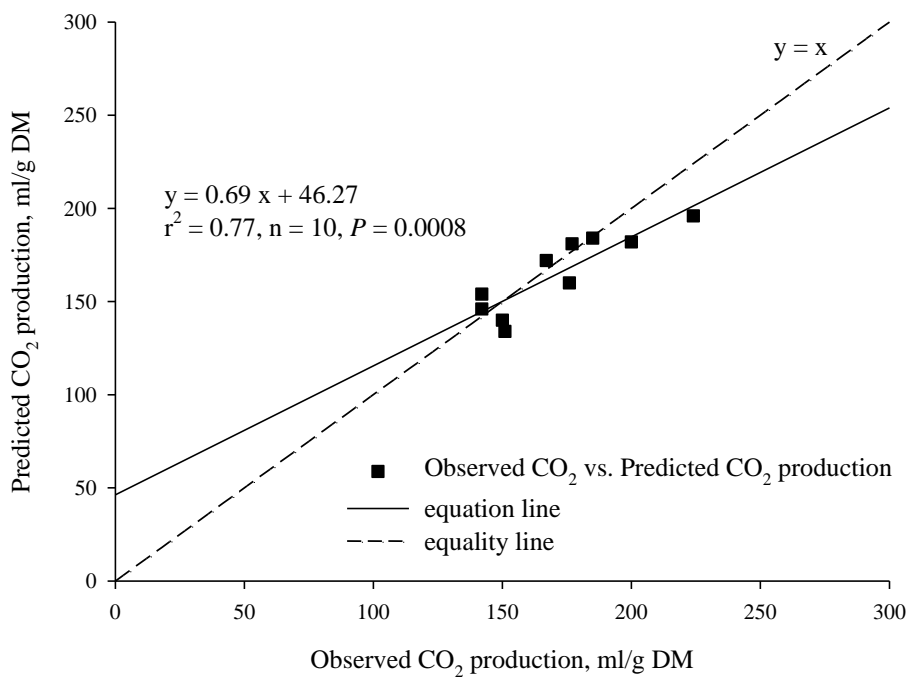

Figure 2. Relationship between the observed vs the predicted $\mathrm{CO}_{2}$ production. 


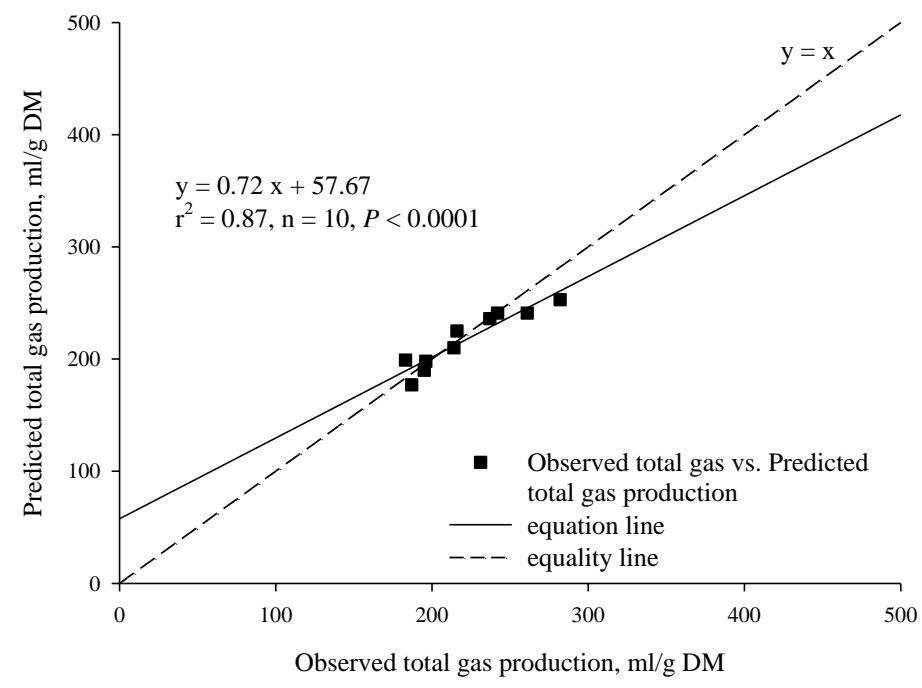

Figure 3. Relationship between the observed vs the predicted total gas production.

production. In the present trial, the $\mathrm{CP}$ content of the mixed rations for modeling was from 6.44 to $15.04 \%$ and that for validation was from 6.50 to $14.20 \%$, which was in a moderate range, it could be believed that the in vitro gas test was suitable for the gas measurements and the results were reliable.

Relationships between the $\mathrm{CH}_{4}, \mathrm{CO}_{2}$ and total gas production and the CNCPS carbohydrate fractions

The CNCPS divides the carbohydrates of feeds for ruminants into four fractions, i.e. $\mathrm{CA}$ (sugar), $\mathrm{CB}_{1}$ (starch and pectin), $\mathrm{CB}_{2}$ (available fibre) and $\mathrm{CC}$ (unavailable fibre) (Sniffen et al., 1992). Fractions $\mathrm{CA}, \mathrm{CB}_{1}$ and $\mathrm{CB}_{2}$ can be fermented in the rumen at fast, moderate and slow speed, respectively, whereas fraction $\mathrm{CC}$ is not fermentable in the rumen. The classification of the CNCPS carbohydrate fractions reflects the carbohydrate composition as well as the fermentative characteristics of the carbohydrates. Significant positive regression relationships found in the present trial between the $\mathrm{CH}_{4}$ production (Equation I), the $\mathrm{CO}_{2}$ production (Equation II), the total gas production (Equation III) and the CNCPS carbohydrate fractions CA, $\mathrm{CB}_{1}$ and $\mathrm{CB}_{2}$ indicated that the CNCPS carbohydrate fractions were suitable parameters for predicting the $\mathrm{CH}_{4}$ production, the $\mathrm{CO}_{2}$ production and the total gas production.

The carbohydrate fraction $\mathrm{CC}$ was not included in the equations because it was screened out by the stepwise regression analysis. The result was in accordance with the fermentative characteristics of fraction CC in the CNCPS (Sniffen et al., 1992). The results were also in agreement with the results of Moe and Tyrell (1979) who found that no significant regression relationship existed between the $\mathrm{CH}_{4}$ production and the lignin intake of Holstein cows.

Ruminal fermentation of cellulose and hemicellulose mainly produces acetate and butyrate accompanied with the formation of $\mathrm{CO}_{2}$ and $\mathrm{H}_{2}$ which are used for $\mathrm{CH}_{4}$ production, whereas fermentation of sugars and NSC mainly produces propionate accompanied with an uptake of $\mathrm{H}_{2}$. It could be presumed that the fermentation of fraction $\mathrm{CB}_{2}$ would produce more $\mathrm{CH}_{4}$ than that of fractions $A$ and $\mathrm{CB}_{1}$. In Equation I, however, the coefficients of $\mathrm{CA}$ and $\mathrm{CB}_{1}, \mathrm{CB}_{2}$ are $89.16,124.10$, and $30.58 \mathrm{~mL} / \mathrm{g}$, respectively, indicating that fraction $\mathrm{CB}_{2}$ produced less $\mathrm{CH}_{4}$ than fractions $\mathrm{CA}$ and $\mathrm{CB}_{1}$. The reason for the results could be that $\mathrm{CB}_{2}$ was the available fibre with slower fermentation rate than fractions $\mathrm{CA}$ and $\mathrm{CB}_{1}$.

Moe and Tyrell (1979) studied the relationship among the diet composition, intake and $\mathrm{CH}_{4}$ production of Holstein cows and found a regression relationship between the $\mathrm{CH}_{4}$ production and the intakes of the soluble residue, hemicellulose and cellulose $\left(\mathrm{R}^{2}=0.67\right)$ and the regression relationship became closer when the apparently digested soluble residue, hemicellulose and cellulose were used as the dietary variables $\left(\mathrm{R}^{2}=0.73\right)$. Jentsch et al. (2007) found a regression relationship between the $\mathrm{CH}_{4}$ production and the intakes of $\mathrm{CP}$, crude fat, crude fibre and $\mathrm{N}$-free extract of cattle $\left(\mathrm{R}^{2}=0.859\right)$. The regression relationship also became closer when the digestible nutrients including $\mathrm{CP}$, $\mathrm{EE}$, crude fibre and $\mathrm{N}$-free extract were used as the dietary variables $\left(\mathrm{R}^{2}=0.896\right)$. In Equation I of the present trial, the determination coefficient $\left(\mathrm{R}^{2}=0.81\right)$ between the $\mathrm{CH}_{4}$ production and the CNCPS fractions $\mathrm{CA}$ and $\mathrm{CB}_{1}, \mathrm{CB}_{2}$ was between the determination coefficient of Moe and Tyrell (1979) and that of Jentsch et al. (2007), indicating that Equation I was reliable for predicting the $\mathrm{CH}_{4}$ production.

\section{Validation of the equations established in the trial}

Validation of Equations I, II, and III in the present trial using 10 rations indicated that the determination coefficient $\left(\mathrm{R}^{2}\right)$ between the observed and the predicted $\mathrm{CH}_{4}, \mathrm{CO}_{2}$ or 
total gas production was high. The RMSPE\% of Equations I, II and III was lower than that of Moe and Tyrrell (1979) (34\%), Ellis et al. (2007) (14.4\%) and Blaxter and Clapperton (1965) (36.5\%). The high determination coefficient and the low RMSPE\% indicate that Equations I, II, and III are reliable for predicting the $\mathrm{CH}_{4} \mathrm{CO}_{2}$ or total gas production of rations with the roughage/concentrate ratios within the range of 10:90, 20:80, 30:70, 40:60, and 50:50 for cattle. The trial demonstrated that the CNCPS fractions $\mathrm{CA}$ and $\mathrm{CB}_{1}, \mathrm{CB}_{2}$ are suitable dietary variables for modeling $\mathrm{CH}_{4}$ production.

It should be noted, however, that Equations I, II, and III in the present trial were developed based on the in vitro measurement of gas production. Since rumen is a dynamic system with the passage and digestion of digesta taking place simultaneously, and the substrate for methanogenesis shifts from hydrogen to formate after $10 \mathrm{~h}$ of in vitro batch culture (Seo et al., 2009), this trial suffers from the limitations of the $48 \mathrm{~h}$ in vitro incubation. The equations need to be validated using in vivo trials for predicting the $\mathrm{CH}_{4} \mathrm{CO}_{2}$ and total gas production from rumen fermentation of cattle.

\section{CONCLUSION}

A significant multiple linear regression relationship was found between the $\mathrm{CH}_{4}$ production and the CNCPS carbohydrate fractions $\mathrm{CA}, \mathrm{CB}_{1}$ and $\mathrm{CB}_{2}$ of rations for cattle over a wide range of concentrate/roughage ratios. Evaluation results demonstrated that the in vitro $\mathrm{CH}_{4}$ production of rations for cattle could be accurately predicted based on the CNCPS carbohydrate fractions CA, $\mathrm{CB}_{1}$ and $\mathrm{CB}_{2}$ using Equation I. To utilize the equation for predicting the $\mathrm{CH}_{4}$ production from rumen fermentation of cattle, it is necessary to validate the equation using in vivo trials.

\section{ACKNOWLEDGEMENTS}

This trial was financially supported by the National Natural Science Foundation of China (Project no. 31072055) and The Ministry of Science and Technology of China (Project no. 2011BAD26B03-4).

\section{REFERENCES}

AOAC. 1990. Official methods of analysis. 15th ed. Arlington (VA): Assoc. Off. Anal. Chem.

Axelsson, J. 1949. The amount of produced methane energy in the European metabolic experiments with adult cattle. Ann. R. Agric. Coll. Sweden 16:404-419.
Blaxter, K. L. and J. L. Clapperton. 1965. Predition of the amount of methane produced by ruminants. Br. J. Nutr. 19:511-522.

Bratzler, J. W. and E. B. Forbes. 1940. The estimation of methane production by cattle. J. Nutr. 19:611-613.

Ellis, J. L., E. Kebreab, N. E. Odongo, B. W. McBride, E. K. Okine, and J. France. 2007. Prediction of methane production from dairy and beef cattle. J. Dairy Sci. 90:3456-3466.

Goering, H. K. and P. J. Van Soest. 1970. Forage fibre analysis. Agric. Handbook No. 379. ARS-USDA, Washington, DC.

Jentsch, W., M. Schweigel, F. Weissbach, H. Scholze, W. Pitroff, and M. Derno. 2007. Methane production in cattle calculated by the nutrient composition of the diet. Arch. Anim. Nutr. 61: 10-19.

Kriss, M. 1930. Quantitative relations of the dry matter of the food consumed, the heat production, the gaseous outgo, and the insensible loss in body weight of cattle. J. Agric. Res. 40: 283295.

Liu, J. X., A. Susenbeth, and K.-H. Südekum. 2002. In vitro gas production measurements to evaluate interactions between untreated and chemically treated rice straws, grass hay, and mulberry leaves. J. Anim. Sci. 80:517-524.

Mills, J. A. N., E. Kebreab, C. M. Yates, L. A. Crompton, S. B. Cammell, M. S. Dhanoa, R. E. Agnew, and J. France. 2003. Alternative approaches to predicting methane emissions from dairy cows. J. Anim. Sci. 81:3141-3150.

Menke, K. H. and H. Steingass. 1988. Estimation of the energetic feed value obtained from chemical analysis and in vitro gas production using rumen fluid. Anim. Res. Dev. 28:7-55.

Moe, P. W. and H. F. Tyrrell. 1979. Methane production in dairy cows. J. Dairy Sci. 62:1583-1586.

Offner, A. and D. Sauvant. 2004. Comparative evaluation of the Molly, CNCPS, and LES rumen models. Anim. Feed Sci. Technol. 112:107-130.

Prasad, C. S., C. D. Wood, and K. T. Sampath. 1994. Use of in vitro gas production to evaluate rumen fermentation of untreated and urea-treated finger millet straw (Eleusine coracana) supplemented with different levels of concentrates. J. Sci. Food Agric. 65:457-464.

Shannak, S., K.-H. Südekum, and A. Susenbeth. 2000. Estimating ruminal crude protein degradation with in situ and chemical fractionation procedures. Anim. Feed Sci. Technol. 85:195-214

Sniffen, C. J., J. D. O'Connor, P. J. Van Soest, D. G. Fox, and J. B. Russell. 1992. A net carbohydrate and protein system for evaluating cattle diets: II. carbohydrate and protein availability. J. Anim. Sci. 70:3562-3577.

Seo, S., Sang C. Lee, S. Y. Lee, J. G. Seo, and Jong K. Ha. 2009. Degradation kinetics of carbohydrate fractions of ruminant feeds using automated gas production technique. Asian-Aust. J. Anim. Sci. 22:356-364.

Van Soest, P. J., J. B. Robertson, and B. A. Lewis. 1991. Methods for dietary fiber, neutral detergent fiber, and nonstarch polysaccharides in relation to animal nutrition. J. Dairy Sci. 74:3583-3597. 